\title{
Menstrual issues for women with intellectual disability
}

\author{
Jane Tracy \\ Director ${ }^{1,3}$ \\ Sonia Grover \\ Associate professor ${ }^{2}$ \\ Sandra Macgibbon \\ Education director ${ }^{1,3}$ \\ ${ }^{1}$ Centre for Developmental \\ Disability Health Victoria \\ Monash Health \\ ${ }^{2}$ University of Melbourne \\ Royal Children's Hospital \\ ${ }_{3}^{3}$ Monash University \\ Melbourne
}

\section{Key words}

contraception,

dysmenorrhoea, intellectual disability, menstruation

Aust Prescr 2016;39:54-7

http://dx.doi.org/10.18773/ austprescr.2016.024

\section{SUMMARY}

The approach to menstrual management in girls with intellectual disabilities should be the same as it is for other girls. Advice may need to be tailored according to the severity of the disability.

Girls who can manage their own toilet hygiene can usually learn to manage their menses independently. They need preparation for the menarche with information appropriate to their level of understanding.

When assessing menstrual problems, it may help to chart any symptoms against the menstrual cycle to confirm that they are related. The management options for problems such as dysmenorrhoea or heavy bleeding are the same as they are for other women.

\section{Introduction}

Girls with intellectual disability usually go through puberty at the same time as those without disabilities. ${ }^{1,2}$ Their level of understanding, however, may present a challenge to learning the skills needed to manage their menses.

\section{Implications of intellectual disability}

Women with intellectual disability may have difficulty with a range of cognitively based tasks (described in DSM-5 $5^{3}$ ) including:

- conceptual understanding and skills (language, literacy, numeracy, reasoning, knowledge, memory)

- social understanding and skills (empathy, social judgement, interpersonal communication, relationships)

- practical understanding and skills (selfmanagement, personal care, money and time management, recreation, organisation, implementation of school and work tasks).

The impact on daily life (adaptive functioning) depends both on the severity of her intellectual disability and on the education, resources and supports available to her.

As a rule of thumb, if a girl can learn to be independent in her toilet hygiene, she can learn to be independent in the management of her menses. For those unable to do so, their menstrual hygiene is managed with continence products and the support of carers, in the same way as bladder and bowel function.

\section{Preparation for menarche}

All girls benefit from preparation before the menarche to understand what is happening in their body. This applies equally to girls with intellectual disabilities. All girls need information provided in ways that are appropriate to their level of understanding.

Girls with intellectual disabilities require clear, direct information, and opportunities to practise new skills. Resources such as the Special Girls' Business picture book are very helpful for them, their families and for professionals working with them (see Box).

\section{Menstrual difficulties}

Girls with intellectual disabilities may take longer to learn the skills required for menstrual management. Problems may reflect a lack of understanding of the practical steps required or appropriate social behaviours. When issues arise, such as blood on clothing, pads put in inappropriate places, or disclosure of private information at inappropriate times or places, the reactions of school staff, other students and parents can be highly charged. The assessment of these difficulties includes ensuring the young woman has the information, support and opportunity to learn and practise the skills she requires to be as independent as possible in her self-care (see Box).

Changes in a young woman's behaviour may be attributed to the menstrual cycle, whether or not this is actually the case. For women not able to clearly express their experiences in words, links between changes in behaviour and the menstrual cycle, which may indicate dysmenorrhoea or premenstrual syndrome, should be confirmed. This can be done by charting behaviour changes over several months in relation to the menstrual cycle. 


\section{Sexual maturity}

Menarche signals reproductive maturity and concerns around menstrual management may become intertwined with concerns around vulnerability to sexual abuse and pregnancy. These are quite separate issues and it is important to explore the presenting problem to clarify the issues and ensure the real underlying concerns are addressed.

Young women who have the capacity to choose to participate in sexual relationships require education around sexual activity, intimate relationships, sexual health and pregnancy. Consideration of contraceptive options is a part of this discussion.

Issues of vulnerability must be addressed to ensure girls and women live free from abuse. This is achieved through education, teaching of protective behaviours and the provision of appropriate environments with adequate social and personal support.

Sexual abuse is a crime. This includes situations in which a woman does not have capacity to consent to sexual activity. When she does not have this capacity and is found to be pregnant or have a sexually transmitted disease, the crime of abuse must have occurred. This should be reported to the police.

\section{Epilepsy and cyclic seizures}

The prevalence of epilepsy is approximately $20-40 \%$ in people with intellectual disability. ${ }^{4}$ In catamenial epilepsy the seizures are linked to the hormonal changes of the menstrual cycle. This link may be suspected by carers, but it is important to confirm the association by charting seizures in relation to the menstrual cycle over several months.

\section{Other medical conditions}

There is a range of medical conditions that can impact on the menstrual cycle. Some genetic syndromes are associated with abnormalities of reproductive function. Prader-Willi syndrome and Laurence-Moon syndrome, for example, are both associated with intellectual disability and hypogonadism. Being underweight from any cause may result in amenorrhoea, while obesity may be associated with heavy or irregular menses. Thyroid dysfunction may also alter menstrual patterns. Drugs associated with menstrual irregularities include anticonvulsants (weight) and antipsychotics (weight and prolactin).

\section{Medical management}

Respect the autonomy, dignity and privacy of all women. Invite the young woman to be seen alone (without parents or carers) for at least part of

\section{Box Resources on menstrual issues for women with} intellectual disabilities

\section{Special Girls’ Business}

A puberty resource picture book written for school-aged girls with special needs and their carers www.secretgb.com/special-girls-business ${ }^{\mathrm{TM}}$

\section{Supporting Women}

Free downloadable resources for GPs and carers:

- Supporting Women: Information and resources for general practitioners supporting women to manage their menstruation http://cddh.monash.org/assets/supporting-women-gp.pdf

- Supporting Women: Information and resources for carers supporting women to manage their menstruation

http://cddh.monash.org/assets/supporting-women-carer.pdf

Sexual and Reproductive Health

Improving sexual and reproductive health for people with disability. Report from the SH\&FPA Disability Special Interest Group. 2013.

www.shinesa.org.au/media/product/2015/04/ImprovingSexualandReproductiveHealth forPeoplewithDisability.pdf

Intellectual disability and healthcare

- Useful information, services and resources for [Victorian] health professionals working with people with developmental disability

www.cddh.monash.org/assets/documents/healthcare-and-people-with-intellectualdisabilities.pdf

- Working with people with intellectual disability in healthcare settings http://www.cddh.monash.org/assets/documents/2015/working-with-people-withintellectual-disabilities.pdf

\section{Legal information}

Contact the relevant state public advocate office for information on consent and disability.

www.advokit.org.au/decision-making/public-advocates/

the consultation. Collect information to confirm the diagnosis of menstrual or cyclic disorders by charting symptoms against the menstrual cycle. If the cycle is irregular then a longer recording period will be required.

If there is no underlying pathology, reassure the woman, educate her about her menstrual cycle and explain the reasons for any symptoms. Suggest simple strategies that may help, including rest, diet, exercise, weight management and relaxation.

\section{Menstrual problems}

First clarify the problem. Is it heavy bleeding or dysmenorrhoea? Quantify by asking how many pads she uses. Possible investigations include a sexually transmitted infection screen (endometritis may increase bleeding, pelvic inflammatory disease may cause pain). Consider ultrasonography if symptoms are persistent or the woman is older. 
Management includes:

- simple non-pharmacological strategies such as warm packs, rest

- $\quad$ simple analgesic (paracetamol) or non-steroidal anti-inflammatory drugs such as mefenamic acid

- non-steroidal anti-inflammatory drugs or tranexamic acid to reduce heavy menstrual loss (30-50\% reduction respectively) ${ }^{5}$

- combined low-dose monophasic oral contraceptive pill which can be used continuously long term, no break being required ${ }^{5}$

- long-acting reversible contraception such as the levonorgestrel-releasing intrauterine system and medroxyprogesterone acetate as these reduce menstrual loss (etonogestrel implants are associated with irregular bleeding in 33\% of women and are therefore not recommended for women with intellectual disability ${ }^{5}$ )

- $\quad$ surgical techniques - these are irreversible and require special court or board approval for a woman with an intellectual disability.

\section{Cyclic behavioural problems}

Communication challenges may make it difficult to distinguish discomfort from mood changes in women who respond to pain with altered behaviour. Management includes:

- explanation and education

- non-pharmacological interventions including physical activity, stress management, healthy lifestyle (diet, exercise, sleep), quitting smoking, low or no alcohol consumption

- trial of the management options for dysmenorrhoea with charting of behaviour to monitor response.

\section{Amenorrhoea}

Primary amenorrhoea may occur if the young woman is underweight, or as a consequence of significant brain injury or disease process, either congenital or acquired. Delayed puberty should be investigated at 14 years and delayed menarche at 16 years, as for young women without a disability.

The most common cause of secondary amenorrhoea is pregnancy. Other causes include weight loss, obesity, pituitary tumours, drugs (e.g. antipsychotics), hyperthyroidism and, rarely, severe emotional disturbance.

\section{Working with carers}

Statistically, most women with intellectual disability have a mild impairment. They can discuss their symptoms and management options with their doctor, as long as adequate time is allowed and the language used is appropriate for their level of understanding Some women require the support of others (family or paid support workers) to access and participate in the consultation and implement management recommendations. Support workers, like most family members, do not have any health training and so clear language (without medical jargon) is required.

Family members usually have an extensive knowledge of the person's social and medical history, personality and function. They are a valuable source of information. Disability workers support people in their personal and community activities. Their training is in working with people with disability, but not in health. The level of knowledge and experience is highly variable. Staff turnover is often high and staff work in shifts. It is therefore important to check how well the person accompanying the woman knows her (regular or casual staff member, length of relationship). Staff will often come with a medical file with information about past history and current health issues, but if more information is required contact the manager or house supervisor.

When implementing a management plan, work in partnership with the person's support network. Write a clear summary of management recommendations. Discuss, explain and write down the key points of diagnosis, investigations, management, expected response and potential adverse effects, and follow up to ensure accurate transmission of information to all involved in care. Review regularly to ensure management recommendations are implemented, expected outcomes are achieved and adverse effects are detected

\section{Legal considerations}

- Capacity to consent should be assessed individually for each decision required. A woman can legally consent if she is able to understand, retain, believe, evaluate, weigh relevant information and express her decision. Capacity to consent is therefore related both to the woman's cognitive ability and to the complexity of the decision (e.g. insertion of an intrauterine device or ablation of endometriotic lesions).

- Most women with intellectual disability have a mild cognitive impairment and can legally consent to treatments for menstrual disorders if information is provided in an accessible format and language.

- Women who have impaired capacity to consent may require the support of another, for example Person Responsible (Victoria), to make decisions about medical treatment. Paid carers are not able to give consent on behalf of the person they are paid to support. 
- The consent of the Family Court (children) or the relevant state Guardianship Tribunal (adults) is required for major medical procedures such as termination of pregnancy and sterilisation.

\section{Conclusion}

Women with intellectual disability have the same menstrual problems as other women. The starting point for management should therefore be just the same as it would be for another woman of the same age.

Modifications to that strategy may be warranted when tailoring the intervention to the individual's needs and her decision-making capacity. These modifications should be justifiable in terms of being in the woman's best interests, the least restrictive option, and complying with any legal requirements. $\varangle$

Conflict of interest: none declared

\section{REFERENCES}

1. Women's health menstrual disorders. In: Management guidelines: developmental disability. Version 3. Melbourne: Therapeutic Guidelines Limited; 2012.

2. Zacharin M, Savasi I, Grover S. The impact of menstruation in adolescents with disabilities related to cerebral palsy. Arch Dis Child 2010;95:526-30. http://dx.doi.org/10.1136/ adc. 2009.174680
3. American Psychiatric Association. Diagnostic and statistical manual of mental disorders. 5th ed. Arlington (VA): American Psychiatric Assocation; 2013.

4. Bowley C, Kerr M. Epilepsy and intellectual disability J Intellect Disabil Res 2000;44:529-43. http://dx.doi.org/ 10.1046/j.1365-2788.2000.00270.x

5. Grover SR. Gynaecological issues in adolescents with disability. J Paediatr Child Health 2011;47:610-3. http://dx.doi.org/10.1111/j.1440-1754.2011.02163.x

\section{FURTHER READING}

Allen K Contraception - common issues and practical suggestions. Aust Fam Physician 2012;41:770-2.

Grover SR. Menstrual and contraceptive management in women with an intellectual disability. Med J Aust 2002;176:108-10.
Wilkinson JE Cerreto MC. Primary care for women with intellectual disabilities. J Am Board Fam Med 2008;21:215-22. http://dx.doi.org/10.3122/jabfm.2008.03.070197

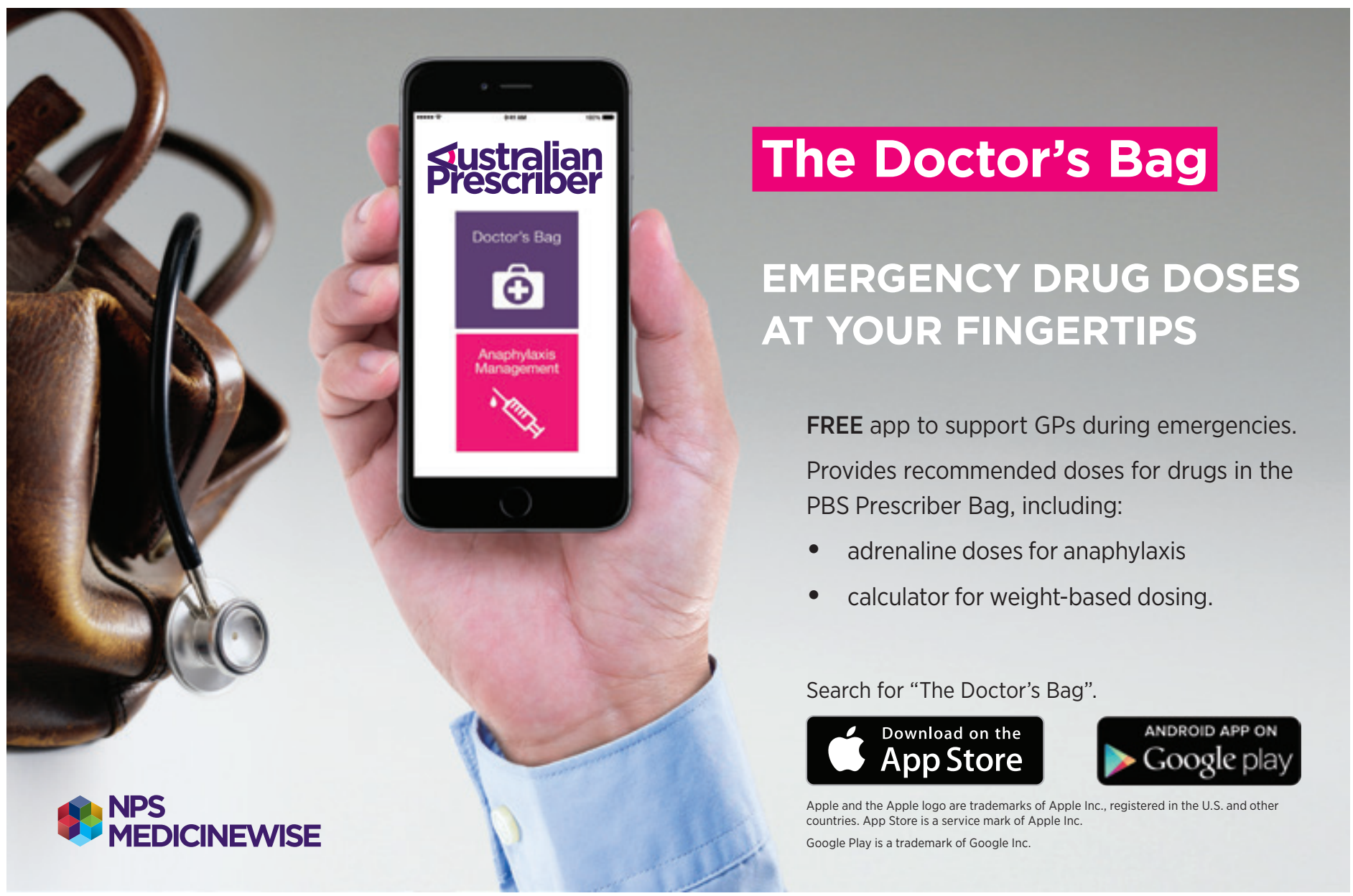

